\title{
Zusammenfassung und Schlusswort
}

Wir befinden uns in einer aufregenden Zeit für die Erforschung der grundlegenden Naturgesetze - einerseits gibt es eine ganze Reihe ungeklärter Fragen, die auf ihre Beantwortung durch neue Experimente warten, und viele Ideen, wie die Antworten aussehen könnten. Andererseits steht jetzt mit dem Large Hadron Collider das leistungsfähigste Instrument zur Verfügung, das die Teilchenphysik je hatte. Dennoch ist bisher unklar, wohin die Reise geht. Die Jahre 2016-2017 könnten zu einem entscheidenden Wendepunkt für die Disziplin werden, sind aber zumindest psychologisch sehr wichtig: Da nach den technischen Problemen von 2015 nun erstmals große Datenmengen bei einer Rekordenergie von $13 \mathrm{TeV}$ gesammelt werden können, geht es jetzt ums Ganze - kann das Standardmodell zu Fall gebracht werden? Wenn nicht, steht die Teilchenphysik vor einem Problem und müsste bis auf Weiteres auf die kleineren laufenden Experimente hoffen, die beispielsweise nach der Dunklen Materie suchen. Eine Entdeckung am LHC hingegen würde die Grundlagenphysik in einem in den letzten Jahrzehnten ungekannten Maß revolutionieren und völlig neue Türen für die zukünftige Forschung aufstoßen.

Ich hoffe, dass ich Ihnen mit den beiden Essentials zur „Physik hinter der Weltmaschine" einen informativen und unterhaltsamen Einblick in die verschiedenen Ideen und Zusammenhänge geben konnte, die hinter diesen Entwicklungen stehen. Nun heißt es abwarten, was die Natur für uns bereit hält! 